\title{
On the properties of strange modes
}

\author{
Hideyuki Saio, ${ }^{1}$ Norman H. Baker ${ }^{2}$ and Alfred Gautschy ${ }^{3}$ \\ ${ }^{1}$ Astronomical Institute, School of Science, Tohoku University, Sendai 980-8578, Japan \\ ${ }^{2}$ Department of Astronomy, Columbia University, New York, NY 10027, USA \\ ${ }^{3}$ Astronomisches Institut, Universität Basel, Venusstrasse 7, CH-4102 Binningen, Switzerland
}

Accepted 1997 September 15. Received 1997 September 8; in original form 1997 July 1

\begin{abstract}
A B S T R A C T
Properties of the so-called strange modes occurring in linear stability calculations of stellar models are discussed. The behaviour of these modes is compared for two different sets of stellar models, for very massive zero-age main-sequence stars and for luminous hydrogendeficient stars, both with high luminosity-to-mass ratios. We have found that the peculiar behaviour of the frequencies of the strange modes with the change of a control parameter is caused by the pulsation amplitude of a particular eigenmode being strongly confined to the outer part of the envelope, around the density inversion zone. The frequency of a strange mode changes because the depth of the confinement zone changes with the control parameter. Weakly non-adiabatic strange modes tend to be overstable because the amplitude confinement quenches the effect of radiative damping. On the other hand, extremely non-adiabatic strange modes become overstable because the perturbation of radiation force (gradient of radiation pressure) provides a restoring force that can be out of phase with the density perturbation. We discuss this mechanism by using a plane-parallel two-zone model.
\end{abstract}

Key words: stars: chemically peculiar - stars: oscillations - stars: variables: other.

\section{INTRODUCTION}

Strange modes of stellar pulsation were discovered in numerical studies some 20 years ago (Wood 1976). Since then pulsationally unstable strange modes have been found in very different classes of stellar models. Examples are He stars [Saio \& Jeffery 1988; Saio, Wheeler \& Cox 1984 (in these papers unstable strange modes were identified as ordinary modes); Gautschy \& Glatzel 1990; Gautschy 1995; Saio 1995], low-mass supergiants (Aikawa 1985; Worrell 1986; Zalewski 1992; Gautschy 1992a), massive main-sequence stars (Gautschy 1992b; Glatzel \& Kiriakidis 1993a; Kiriakidis, Fricke \& Glatzel 1993; Papaloizou et al. 1997) and evolved massive stars (Glatzel \& Kiriakidis 1993b), central stars of planetary nebulae (Gautschy 1993), WR stars (Glatzel, Kiriakidis \& Fricke 1993; Kiriakidis, Glatzel \& Fricke 1996) and classical Cepheids (Buchler, Yecko \& Kolláth 1997). It seems likely that the strange mode phenomenon is responsible for the variability of at least some of these stars. Recently, Glatzel (1994), Papaloizou et al. (1997) and Buchler et al. (1997) discussed analytically the origin of the strange modes. Despite the large number of theoretical studies, many of the properties of strange modes have still been puzzling. In this paper we try to clarify some of the properties of strange modes, referring to numerical results for very massive zero-age main-sequence (ZAMS) models and luminous helium stars.

The concept of a modal diagram has proved to be a powerful tool in describing the pulsational behaviour of a sequence of stellar models. A linearized stability analysis gives a set of (complex) eigenfrequencies $\omega=\left(\omega_{R}, \omega_{I}\right)$, called the eigenspectrum $\Sigma$ of the model. A series of stellar models is constructed by changing a control parameter, $\mathcal{P}$, such as mass or effective temperature. We shall assume that a continuous change of $\mathcal{P}$ leads to a continuous change in the eigenfrequencies $\{\omega(\mathcal{P})\} \equiv \Sigma(\mathcal{P})$. A diagram showing the run of some of the eigenfrequencies as a function of the control parameter is called a modal diagram.

The adiabatic approximation for radial pulsations describes the simplest possible oscillating system and has well-understood mathematical properties (see, e.g., Beyer 1995). If the object is dynamically stable, then all eigensolutions are neutrally stable $\left(\omega_{I} \equiv 0\right)$, and the eigenvalues are well separated and ordered with respect to the number of nodes of their eigenfunctions (Sturm's theorem). The situation is similar to standing acoustic waves confined in a cavity. As the control parameter $\mathcal{P}$ changes, the cavity for the oscillation modes deforms due to the change of the stellar structure, and the associated eigenspectrum deforms accordingly. In classical pulsators such as Cepheids and RR Lyrae stars the adiabatic modal diagrams exhibit a slow variation of almost equally spaced eigenfrequencies as a function of $\mathcal{P}$. Non-adiabatic modal diagrams (for low-order modes) are very similar to the adiabatic ones, because the heat exchange between acoustic waves and the stellar material is small. For this reason also, the oscillation periods derived from a linear adiabatic pulsation analysis of the stellar envelope agree well with the observed ones.

However, drastically different situations are known to exist: Wood (1976) investigated luminous helium stars and found the 
adiabatic and non-adiabatic modal diagrams to differ strongly, showing the adiabatic approximation to be a very poor one for these stars. These objects have luminosity-to-mass ratios of the order of $10^{4}$ in solar units and in much of the envelope the thermal time-scale is comparable with the mechanical one, so that heat can leak efficiently. The modal diagrams contained modes without counterparts in the adiabatic case. As the control parameter $\left(T_{\text {eff }}\right.$ in this case) was changed, the trend of the oscillation frequencies of these additional modes was completely different from that of the regular modes (i.e., those with counterparts in the adiabatic analysis). The additional modes could cross several of the regular ones, and Wood found that they are always strongly damped. The term 'strange modes' was introduced by Cox et al. (1980) when they again tried to characterize these additional modes by comparison with adiabatic modal diagrams. This comparison, however, did not lead to a deeper understanding of the modes. In an extensive study of helium-star pulsations, Saio et al. (1984) identified three different types of strange modes, all of which they believed to be related to thermal waves. Again, all three types of modes were always found to be damped.

The existence of strange modes not associated with thermal waves was first shown by Gautschy \& Glatzel (1990) in their survey of linear radial non-adiabatic eigenmodes in luminous hydrogen-deficient carbon $(\mathrm{HdC})$ stars. The non-adiabatic reversible (NAR) approximation, in which the time derivative of the specific entropy is neglected in the equation for the conservation of thermal energy, was introduced in their study. This approximation corresponds to the limit of extreme non-adiabaticity with diminishing thermal time, and suppresses the thermal mode spectrum. Their objective was to clarify the nature of the modes and to follow unambiguously the 'unfoldings' of the mode interactions, as well as to identify the origin of the strange modes. Calculating the modal diagrams for the HdC stars, Gautschy \& Glatzel (1990) showed that strange modes occur even in the NAR approximation and that they become unstable at the crossing of regular and strange modes. Gautschy \& Glatzel also showed that the instability in the full nonadiabatic analysis is caused by an unstable strange mode. The instability remained of comparable strength even in the NAR approximation - where the classical $\kappa$-mechanism can no longer work - so that an alternative physical explanation was needed. However, a satisfactory physical picture of the mode interaction instabilities has so far eluded our grasp.

Recently, adiabatic strange modes were discovered in stellar pulsation calculations using the newly available opacity tables (Kiriakidis et al. 1993; Gautschy 1993). Sequences of envelopes for very different stellar models showed that besides the slowly varying adiabatic eigenfrequencies, rapid (i.e. over small changes of $\mathcal{P}$ ) variations of the oscillation frequencies were occurring. This behaviour is similar to that of the 'avoided crossings' of $p$ and $g$ modes in the adiabatic non-radial pulsation problem (see e.g. Unno et al. 1989, section 17.1). Hence, even in the adiabatic pulsation problem we now have examples of modal diagrams that at least look like those non-adiabatic ones that contain strange modes. In the radial pulsation problem, only one physical mode family, the acoustic modes, comprises the eigenspectrum $\Sigma$. Kiriakidis et al. argued that due to a sound speed inversion, which is induced by the strong iron bump appearing in the new opacity tables, sound waves are partially reflected. If the reflection is efficient enough the effect is to split the original cavity for the acoustic modes. This is discussed in Section 3.1.

In the present paper we discuss strange modes as understood by Gautschy \& Glatzel (1990), which are not related to a thermal mode. The high model density in the modal diagrams of Gautschy $\&$ Glatzel showed that the strange modes do not simply cross the regular modes, but that a mode interaction occurs with an accompanying 'unfolding' of the crossings. Two different forms of unfoldings were found. The two converging modes may avoid each other in their oscillation frequencies (avoided crossings, as mentioned already in the previous paragraph). The other possibility is that the two modes indeed coalesce, forcing one of the participating modes to become overstable and the other to become stable. The real parts of the frequencies of the two interacting modes are essentially identical and their imaginary parts have approximately the same magnitude but opposite signs. The range in $\mathcal{P}$ over which mode interaction occurs is called an instability band. In the NAR approximation the imaginary parts are of equal magnitude, but in a fully non-adiabatic analysis the mirror-image behaviour is only approximate because the modes are affected by radiative damping. The imaginary part of a strange mode eigenvalue tends to be very large compared with that of an ordinary mode.

The strange modes can be interpreted, at least phenomenologically, as one or more additional oscillation spectra which are superimposed on the regular spectrum (as found, e.g., in the adiabatic analysis). At those frequencies at which members of two spectra are to cross, unfoldings occur into either avoided crossings or instability bands. Despite this picture, which describes the calculated modal diagrams, it remains unclear what the physical mechanism is that decides between the occurrence of one or the other of the possibilities for unfolding. Zalewski (1992) emphasized the importance of the choice of the outer boundary conditions for the emerging strange mode spectrum. He attributed strange modes to the presence of a sufficiently strong entropy peak in an ionization zone and believed the strange modes to be impulse responses to the strong entropy perturbation.

The structure of this paper is as follows: Section 2 describes the two sequences of stellar models that were analysed for their strange mode content. The origin of the strange modes in the two model sequences is investigated in Section 3. Section 4 contains our proposal for the mechanism behind the instability bands.

\section{NUMERICAL MODELLING}

The radial, non-adiabatic pulsation analyses of this study were performed on two sequences of stellar models in complete equilibrium. The first is a linear series of chemically homogeneous main-sequence models using OPAL opacities based on the intermediate coupling scheme (Iglesias, Rogers \& Wilson 1992). The control parameter is the mass, which ranges from 50 to $118 \mathrm{M}_{\odot}$. The chemical composition was assumed to be $X=0.7, Y=0.27$. The second series is essentially that of the hydrogen-deficient carbon star (HdC) model envelopes used in the study of Gautschy \& Glatzel (1990), in which old Los Alamos opacity tables were used. The parameters of the envelopes are appropriate for stars of $0.9 \mathrm{M}_{\odot}$ at a luminosity of $\log L / \mathrm{L}_{\odot}=4.05$. The control parameter is $\log T_{\text {eff }}$, ranging from 3.8 (model 1) to 4.3 (model 35). The envelopes are homogeneous with $X=0.0, Y=0.903, X_{\mathrm{C}}=0.081$ and $X_{\mathrm{O}}=0.010$.

Fig. 1 shows the structure of the outer parts of the $80-\mathrm{M}_{\odot}$ $\left(\log L / \mathrm{L}_{\odot}=5.93, \log T_{\text {eff }}=4.672\right)$ main-sequence model and the fifth HdC model ( $\left.\log T_{\text {eff }}=3.859\right)$. Various quantities are plotted as functions of the fractional radius $r / R$. The bottom panels show runs of pressure $P$, density $\rho$, temperature $T$, and the logarithmic pressure gradient $V \equiv-\mathrm{d} \ln P / \mathrm{d} \ln r$. In both models there are density inversions at the peaks of opacity which are due to helium ionizations in 

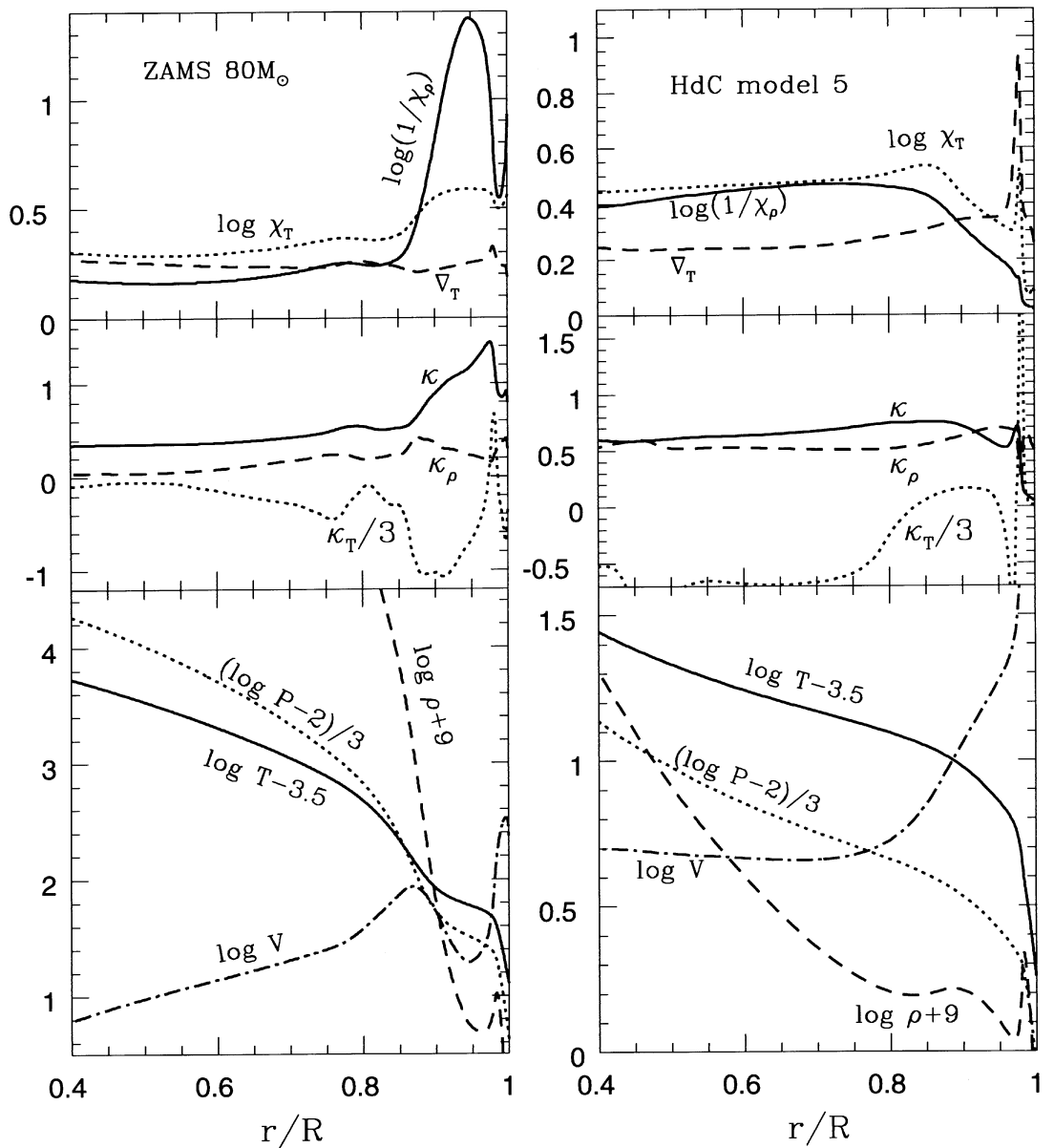

Figure 1. Some physical variables versus fractional radius for an $80-\mathrm{M}_{\odot}$ main-sequence model (left) and the fifth model of our HdC model series (right). Variables displayed are are pressure $P$, temperature $T$, density $\rho, V \equiv-\mathrm{d} \ln P / \mathrm{d} \ln r$, opacity $\kappa$, and its derivatives $\kappa_{T} \equiv(\partial \ln \kappa / \partial \ln T)_{\rho}$ and $\kappa_{\rho} \equiv(\partial \ln \kappa / \partial \ln \rho)_{T}$, temperature gradient $\nabla_{T}=\mathrm{d} \ln T / \mathrm{d} \ln P$, and the pressure derivatives, $\chi_{\rho} \equiv(\partial \ln P / \partial \ln \rho)_{T}$ and $\chi_{T} \equiv(\partial \ln P / \partial \ln T)_{\rho}$.

HdC models and Fe lines at $T \sim 2 \times 10^{5} \mathrm{~K}$ (Iglesias et al. 1992) in the massive ZAMS models. Runs of opacity and its derivatives

$\kappa_{T}=\left(\frac{\partial \ln \kappa}{\partial \ln T}\right)_{\rho}$ and $\kappa_{\rho}=\left(\frac{\partial \ln \kappa}{\partial \ln \rho}\right)_{T}$

are given in the middle panels. Since peaks in opacity make the temperature gradient $\nabla_{T}$ (top panels) high, density inversions are located in convection zones. Convection was treated according to standard mixing-length theory, assuming a mixing length of 1.5 pressure scaleheights $\left(H_{\mathrm{p}}\right)$. The fraction of energy carried by convection is negligibly small in HdC models. It is less than 20 per cent in the envelopes of the massive ZAMS models. The effect of perturbed convective flux is disregarded in our stability analyses.

The uppermost panels show, in addition to $\nabla_{T}$, runs of pressure derivatives,

$\chi_{\rho} \equiv(\partial \ln P / \partial \ln \rho)_{T} \quad$ and $\quad \chi_{T} \equiv(\partial \ln P / \partial \ln T)_{\rho}$,

both of which are unity for the ideal gas law. These quantities deviate from unity significantly in most parts of the models, which is mainly caused by the importance of radiation pressure. For fully ionized gas $\chi_{\rho} \simeq \beta$ and $\chi_{T} \simeq 4-3 \beta$, with $\beta$ being the ratio of gas pressure to total pressure.

The linear differential equations for the radial pulsation were solved by a finite difference method. The mechanical inner and outer boundary conditions adopted are $\partial(\xi / r) / \partial r=0$ and
$\partial(\Delta P / P) / \partial r=0$, respectively, where $\xi$ is the displacement from equilibrium position and $\Delta P$ the Lagrangian pressure perturbation. The thermal inner and outer boundary conditions are $\Delta L=0$ and $\Delta L / L=2 \xi / R+4 \Delta T / T$, respectively.

For both series of models, we have obtained eigenfrequencies under the adiabatic and the NAR approximations, in addition to full non-adiabatic analyses. As stated in Section 1, the NAR approximation is the limit of extreme non-adiabaticity, in which the luminosity perturbation vanishes. Fig. 2 shows our numerical results for adiabatic (uppermost panels), full non-adiabatic (middle panels) and NAR (lowest panels) analyses. We call each panel a modal diagram, in which the vertical axis shows the real part of the angular frequency of pulsation (normalized by $\sqrt{G M / R^{3}}$ ) and the horizontal axis is a control parameter $\mathcal{P}$ (the total mass for ZAMS models and effective temperature for HdC models). In this figure, crosses represent purely real frequencies (neutrally stable) for the adiabatic and for NAR results, and frequencies with positive imaginary parts (stable) for the non-adiabatic results. Filled circles indicate overstable modes with negative imaginary parts. Under the NAR approximation complex eigenfrequencies always have complex conjugate counterparts; i.e., each filled circle represents two modes forming a complex conjugate pair.

As strange modes we identify those eigenfrequency branches that behave differently from those that change only very slowly under a change of the control parameter. We will consider strange modes 

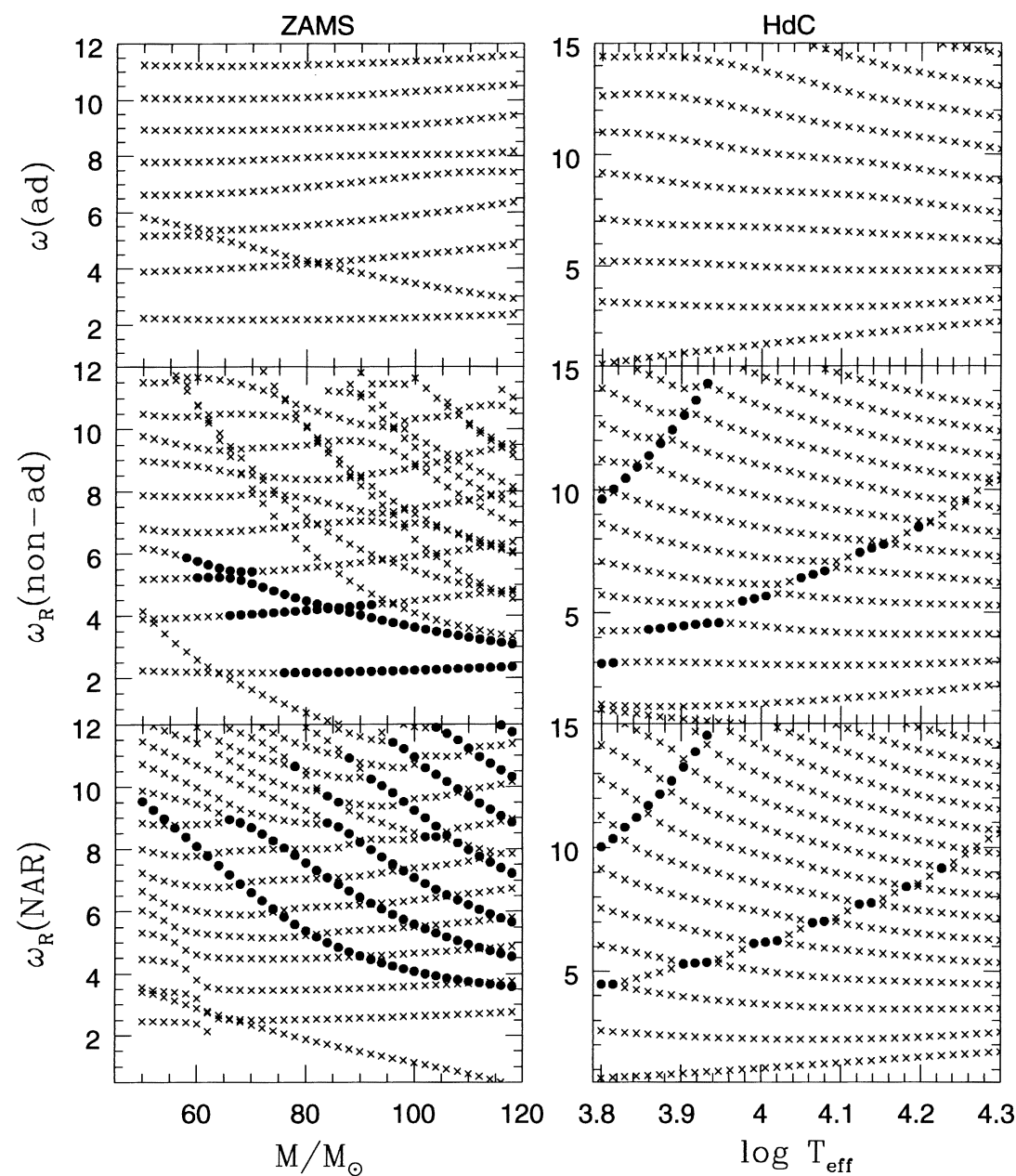

Figure 2. Modal diagrams for massive main-sequence stars (left) and HdC stars (right) under the adiabatic (uppermost panels) and NAR (lowest panels) approximations, and for full non-adiabatic analyses (middle panels). The vertical axes present the oscillation frequency normalized by $\sqrt{G M / R^{3}}$. The horizontal axes, which represent control parameters, are the stellar mass (solar unit) for the main-sequence stars and the logarithm of the effective temperature for HdC stars. Crosses represent modes with vanishing imaginary part of the eigenfrequency for the adiabatic and NAR approximations and modes with positive imaginary part (damping oscillation) for full non-adiabatic analysis. Filled circles represent modes with negative imaginary part for full non-adiabatic analysis and with finite imaginary parts for the NAR approximation, in which if there is a complex frequency, its complex conjugate exists.

that appear in the adiabatic modal diagram as well as those in the NAR modal diagram. We will show below that the rapid frequency change of these strange modes is attributed to a confinement of pulsation amplitude to a thin zone near the surface.

As is shown below, the NAR approximation for the ZAMS models is not a good approximation for the full non-adiabatic pulsations in these models. It is instructive, however, to consider the NAR limit in these models, because pulsations in WR star models, for example, are extremely non-adiabatic and the character of the strange modes in the modal diagram is similar to that for the NAR limit of the ZAMS models (Glatzel et al. 1993).

In general, the discussion of the full non-adiabatic modal diagrams is further complicated because they contain, in addition to the strange modes defined above, modes essentially of thermal origin, whose eigenfrequencies have large positive imaginary parts (i.e., they are strongly damped). In the non-adiabatic analyses for HdC models, however, we did not pick up such thermal modes. We note that if OPAL opacity data - with its Z-bump - were used to model HdC stars, the resulting modal diagram would be more complex (cf. Gautschy 1995; Saio 1995).

\section{THE ORIGIN OF THE STRANGE MODES}

In this section, with various numerical experiments and some analytical considerations, we try to elucidate the very occurrence of strange modes in the modal diagrams.

\subsection{Adiabatic strange modes}

As seen in the top diagram of Fig. 2 for massive ZAMS models, strange modes can also be found in adiabatically treated pulsations. This was also found by Kiriakidis et al. (1993) and Gautschy (1993). We discern two modal sequences, both of them showing pulsation frequencies decreasing with mass. The lower strange mode starts around $\omega=6$ at $50 \mathrm{M}_{\odot}$ and decreases through a series of avoided crossings to about $\omega=3$ at $118 \mathrm{M}_{\odot}$. (Throughout this paper we use the following convention: the symbol $\sigma$ with or without subscripts stands for pulsation frequency with its physical dimension, and $\omega$ stands for the frequency normalized by $\sqrt{G M / R^{3}}$.) The second strange mode at higher frequencies manifests itself only by a broad avoided crossing around $\omega=8$ above about $100 \mathrm{M}_{\odot}$. For a given model the ordering of the number of 


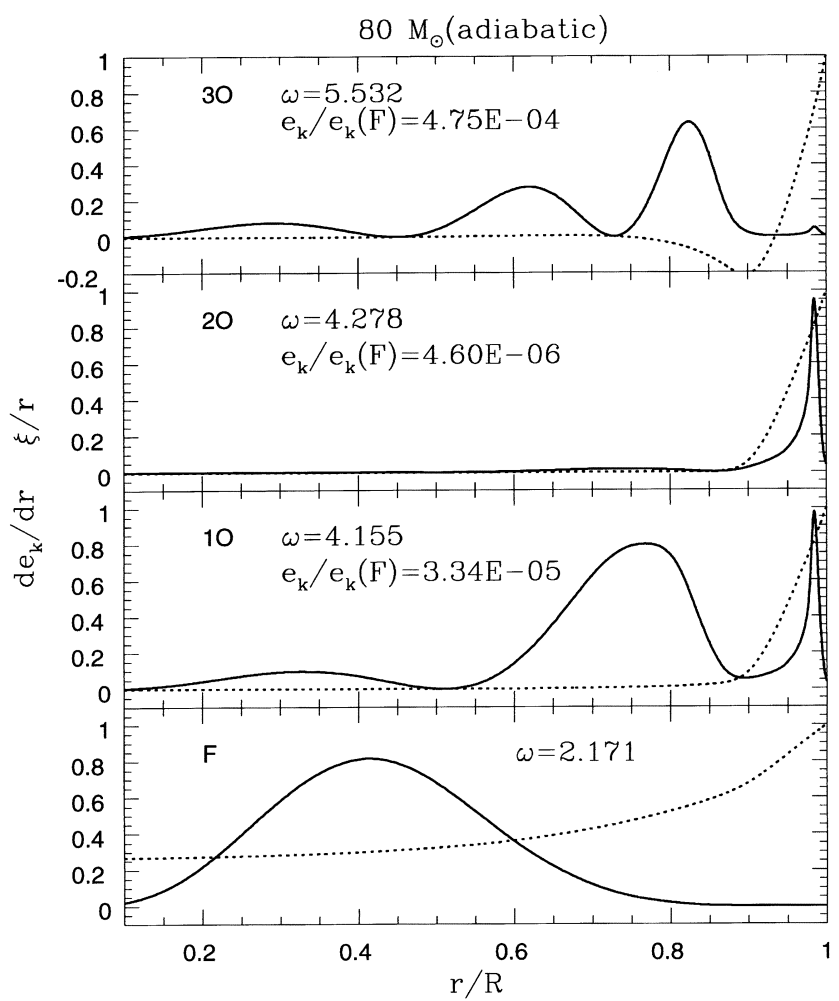

Figure 3. The distribution of pulsation (kinetic) energy density $\mathrm{d} e_{\mathrm{k}} / \mathrm{d} r \propto \omega^{2} \xi^{2} \rho r^{2}$ (solid curves) and the radial displacement (broken lines) $\xi$ are shown against the fractional radius $r / R$ for the four lowest adiabatic modes in an $80-\mathrm{M}_{\odot}$ ZAMS model. Also shown in each panel of overtone modes is the pulsation energy relative to the fundamental mode. In this model the pulsation energy of $2 \mathrm{O}$, which is on the peculiar sequence on the modal diagram, is strongly confined in the outer part of the envelopes.

nodes with respect to frequency is always maintained despite the occurrence of mode crossings. The lower adiabatic strange mode for $M / \mathrm{M}_{\odot}>84$ is identified as the first overtone (1O) and for $62<M / \mathrm{M}_{\odot}<80$ as the second overtone (2O), etc. (The adiabatic pulsation equations admit real eigenvalues only. Therefore, mode interactions can only appear as avoided crossings.)

The different natures of strange modes from ordinary modes may be seen in the spatial distribution of their pulsation energy. Fig. 3 shows $\mathrm{d} e_{\mathrm{k}} / \mathrm{d} r \equiv \omega^{2} \xi^{2} \rho r^{2}$, which is proportional to the local kinetic energy density of the pulsation, plotted versus $r / R$ for several modes of the $80-\mathrm{M}_{\odot}$ ZAMS model. Also shown is the pulsation energy $e_{\mathrm{k}}$ normalized by that of the fundamental mode. The kinetic energy of the strange mode ( $2 \mathrm{O}$ in this model) is strongly confined to the outermost layers and essentially vanishes in layers interior to the density minimum. Hence, only very superficial regions of the star determine the strange mode, very much in contrast to the other modes shown in Fig. 3 (F, 1O, 3O). This causes strange modes to have small kinetic energy compared with regular modes and reduces radiative dissipation in the interior. Both effects tend to destabilize strange modes in the weakly non-adiabatic condition.

In the following we study the origin of the trapping of the amplitude of radial adiabatic pulsation modes with the help of a local analysis as it is known from non-radial pulsation theory. The equation for radial adiabatic pulsations (Cox 1974) can be written as $\frac{\mathrm{d}^{2} y}{\mathrm{~d} r^{2}}+\mathcal{L} y=0$
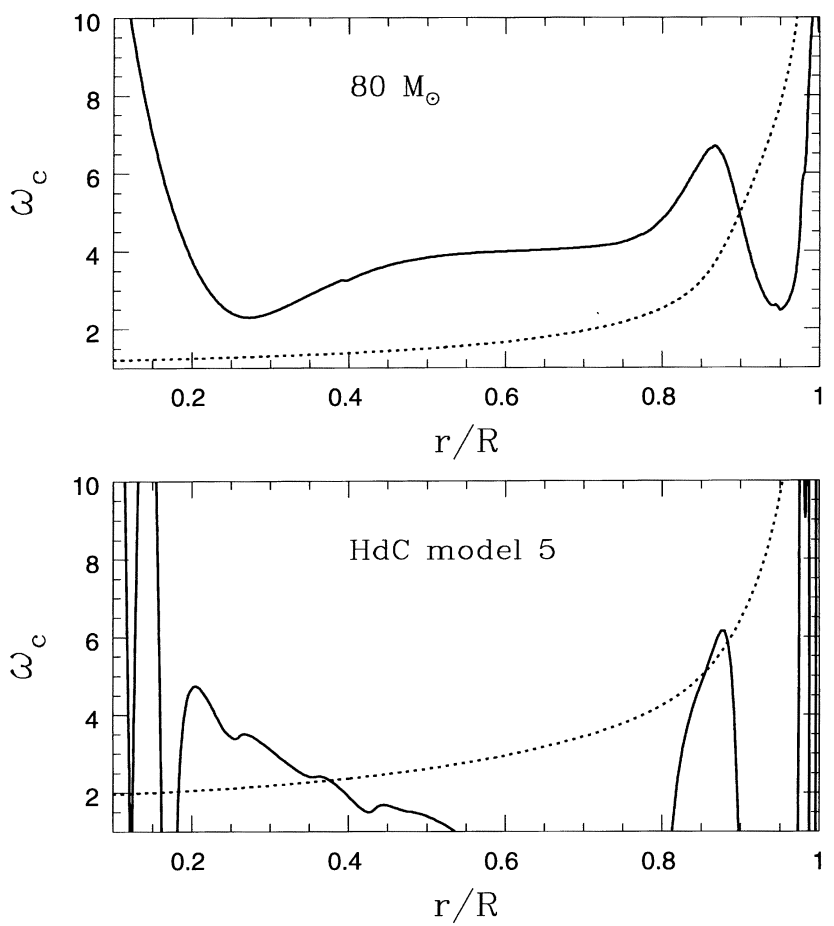

Figure 4. The adiabatic local critical frequency (solid curves), above which radial oscillations are propagative, versus fractional radius $r / R$. The meaning of the dotted lines is explained in the text.

with

$$
\begin{aligned}
\mathcal{L}= & \frac{\sigma^{2} \rho}{\Gamma_{1} P}+\frac{1}{\Gamma_{1} P r} \frac{\mathrm{d}}{\mathrm{d} r}\left[\left(3 \Gamma_{1}-4\right) P\right] \\
& -\frac{1}{2 \sqrt{\Gamma_{1} P r^{4}}} \frac{\mathrm{d}}{\mathrm{d} r}\left[\frac{1}{\sqrt{\Gamma_{1} \operatorname{Pr}^{4}}} \frac{\mathrm{d}}{\mathrm{d} r}\left(\Gamma_{1} P r^{4}\right)\right],
\end{aligned}
$$

where $y \equiv \sqrt{\Gamma_{1} P r^{4}} \xi / r$ with $\xi$ representing the displacement, whose temporal dependence is assumed to be proportional to exp(i $\sigma t)$. We assume the solution to be locally of the form $y \propto \exp [\mathrm{i} k(r) \cdot r]$. Such a local ansatz is a reasonable approximation for short-wavelength modes only, but it should provide us at least with a guiding picture of the spatial behaviour of the pulsation modes even in the case of the lowest order modes. We define $\sigma_{\mathrm{c}}(r)$ as the local critical frequency for which $\mathcal{L}=0$. A perturbation with oscillation frequency $\sigma$ is propagative in regions where $\sigma>\sigma_{\mathrm{c}}$, while the amplitude, $y$, varies exponentially in the evanescent regions where $\sigma<\sigma_{\mathrm{c}}$.

It can be shown that $\sigma_{\mathrm{c}} \simeq \Gamma_{1} g / 2 c_{\mathrm{s}}=c_{\mathrm{s}} / 2 H_{\mathrm{p}}$ in the stellar envelope if the spatial variation of $\Gamma_{1}$ is small, where $g$ is local gravity, $c_{\mathrm{s}}$ adiabatic sound velocity, $H_{\mathrm{p}}$ pressure scaleheight. This equation indicates that the oscillation is propagative when the period is shorter than the time-scale for hydrostatic adjustment $\left(H_{\mathrm{p}} / c_{\mathrm{s}}\right)$. The critical frequency is lower when the sound speed is higher for a given gravity.

Fig. 4 shows the spatial variations of the normalized critical frequency $\omega_{\mathrm{c}}$ (solid curves) for the $80-\mathrm{M}_{\odot}$ model and the fifth $\mathrm{HdC}$ model. The critical frequency in the $80-\mathrm{M}_{\odot}$ model has a minimum at $r / R \simeq 0.95$ (just interior to the density minimum). In the outer part of the envelope $\omega_{\mathrm{c}}$ is approximately proportional to $\sqrt{V}$ $\left(\propto \sqrt{g} / c_{\mathrm{s}}\right.$ ). (In the HdC model, the spatial variation of the adiabatic exponent $\Gamma_{1}$ in the helium ionization zones affects $\omega_{\mathrm{c}}$ significantly.) The drop of the critical frequency is caused by a peak in the sound 


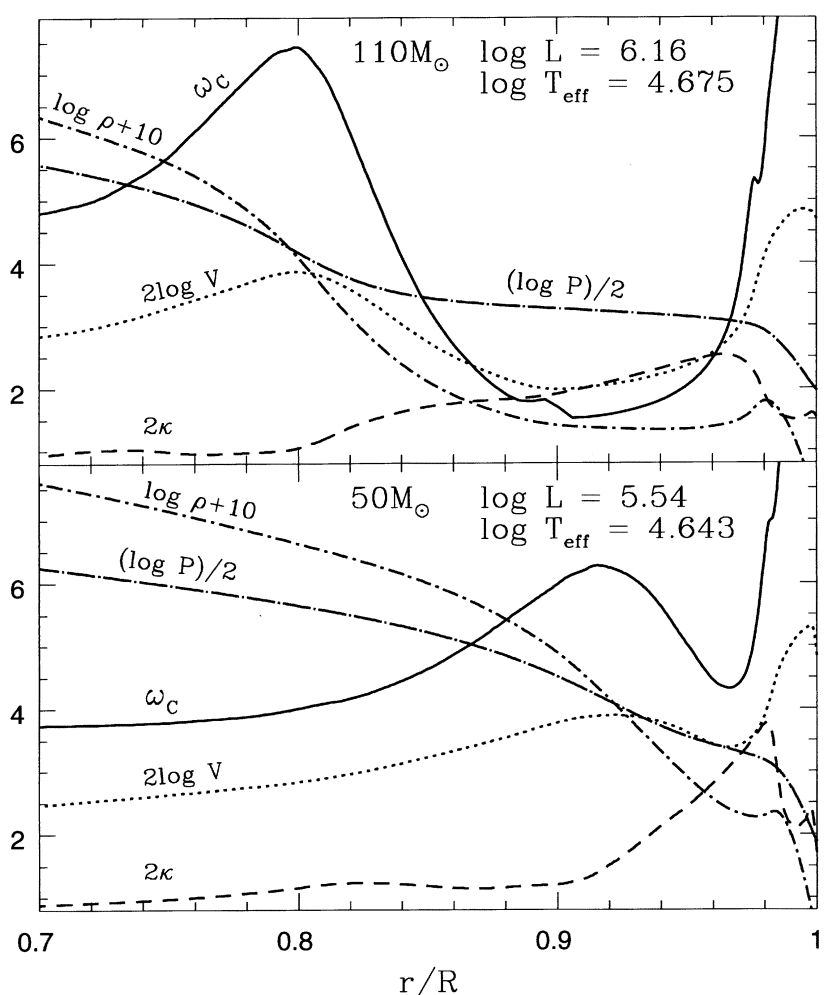

Figure 5. Runs of critical frequency and other quantities in the outer parts of the $50-\mathrm{M}_{\odot}$ (lower panel) and $110-\mathrm{M}_{\odot}$ ZAMS models.

velocity (cf. Gautschy 1993; Kiriakidis et al. 1993). The 'valley' of $\omega_{c}$ can trap a low-frequency pulsation mode to produce adiabatic strange modes.

If a standing wave of a given $\omega$ is to be produced in a propagation region, its wavelength must correspond to the extent of the propagation region. That is, the oscillation should naturally have nodes near the transition to an evanescent region. In order to estimate roughly the position of such nodal points we define an angular frequency

$\omega_{\mathrm{t}}(r)=\frac{\pi}{\sqrt{G M / R^{3}} \int_{r}^{R} c_{\mathrm{s}}^{-1} \mathrm{~d} r}$,

which corresponds to the time required for a sound wave to travel from the surface to radius $r$ and back. Runs of $\omega_{t}(r)$ are shown in Fig. 4 by dotted lines. For a given oscillation frequency $\omega$ the quantity $\omega_{\mathrm{t}}\left(r^{*}\right)=\omega$ gives the approximate location $r^{*}$ of the node of displacement that lies closest to the surface. If for a normal mode of frequency $\omega$ the inner boundary of the propagation region occurs at radius $r_{\mathrm{b}}$, and if $r_{\mathrm{b}}$ is close to this $r^{*}$, that mode is the lowest order mode that can be trapped in the outer propagation region. The upper panel of Fig. 4 shows that in the frequency range between 4 and 6 the pulsation amplitude can be confined to an outer zone that includes the density minimum of the ZAMS model. In this frequency range two kinds of pulsations are possible, one being effectively trapped in the outer envelope and the other being mostly confined to the interior; the former is identified as a strange mode. Comparing Fig. 3 with Fig. 4 , we see that $2 \mathrm{O}$ for $80 \mathrm{M}_{\odot}$ is trapped in the outer propagation zone, while the kinetic energy of pulsation for other modes is also large in the deeper propagation region. Since the frequency difference between $1 \mathrm{O}$ and $2 \mathrm{O}$ modes is very small it is seen that a small frequency mismatch destroys the confinement to the exterior regions.

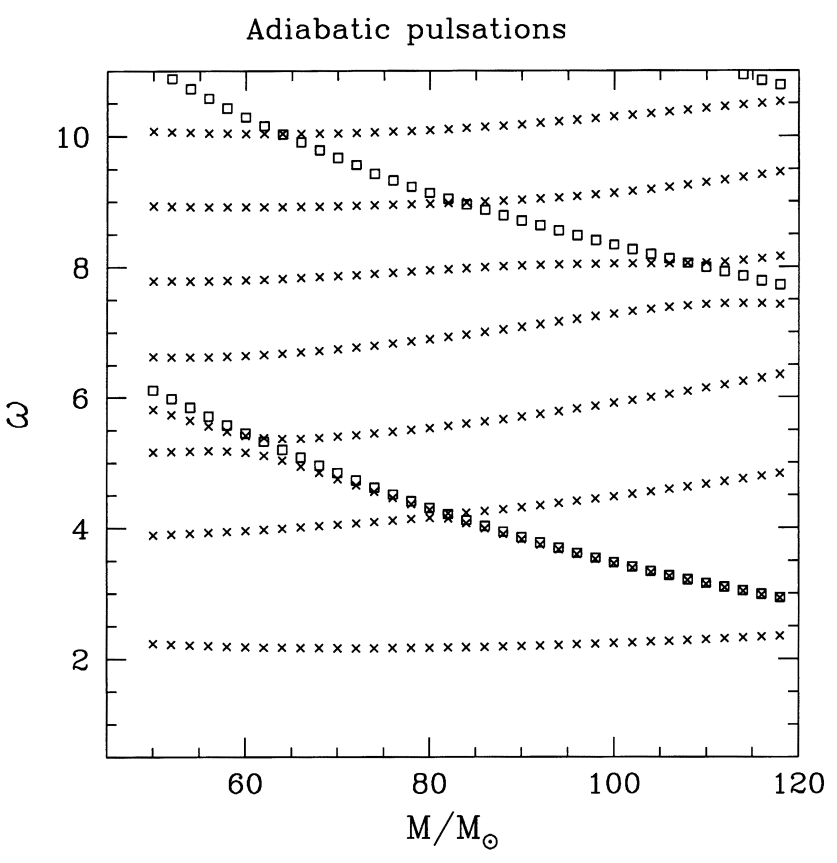

Figure 6. Adiabatic modal diagram for modes obtained by setting the inner boundary condition at $\log P=8$ (open squares) superposed by the true adiabatic modal diagram shown in Fig. 2 for massive main-sequence stars.

Fig. 5 shows runs of critical frequency as well as of some physical quantities in the outer parts of the $50-\mathrm{M}_{\odot}$ and $110-\mathrm{M}_{\odot}$ ZAMS models. This figure shows that as the mass increases the thickness of the outer propagation zone due to the drop of $\omega_{c}$ increases, which is caused by an increase in mass-luminosity ratio with mass. Because the sound travel time in the propagation zone increases with the thickness, the frequency of the lowest order mode trapped in it decreases. This property explains the fact that the normalized frequency of a strange mode decreases as the mass increases.

This interpretation is further supported by constructing models in which the envelope is artificially truncated by placing an inner boundary at $\log P=8$, which is approximately located at the peak of $\omega_{c}$ (see Figs 4 and 5), and computing the frequency of this shallower cavity. The results are shown in Fig. 6 by squares superposed on the adiabatic modal diagram for complete massive ZAMS models. The frequencies resulting from the experiment with the artificial inner boundary trace well the frequencies of the strange modes in the full calculation. Fig. 6 also shows that the small kinks in the regular mode sequences seen near $\omega_{R} \sim 8$ and $M / \mathrm{M}_{\odot} \gtrsim 100$ in the top left diagram of Fig. 2 correspond to the frequencies of the 'first overtone' of the pulsations confined to the outermost regions.

The above discussion clearly shows that the 'valley' in the run of $\omega_{\mathrm{c}}$ is the origin of the adiabatic strange modes. The drop of $\omega_{\mathrm{c}}$ is attributed to the depression of $V(\propto \rho / P)$ or the inversion of sound velocity, which normally decreases outward. Such a phenomenon can be interpreted using the equation of hydrostatic equilibrium. It can be written as

$\frac{\mathrm{d} \ln \rho}{\mathrm{d} r}=-\frac{G M_{r} \rho}{r^{2} P_{\mathrm{G}}}\left[1-\frac{\kappa L}{4 \pi c G M_{r}}\left(1+\frac{P_{\mathrm{G}}}{4 P_{\mathrm{R}}}\right)\right]$

in the stellar envelope where most of the energy is carried by 


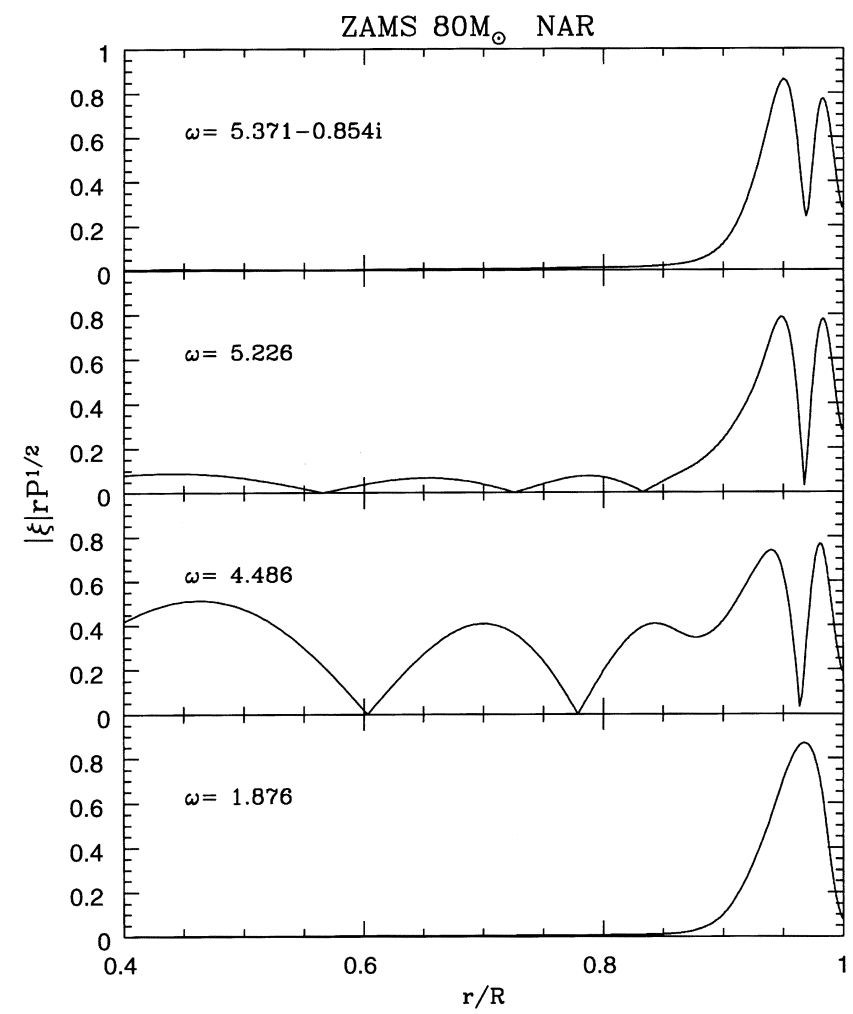

Figure 7. Runs of the amplitude of displacement in the NAR approximation for selected modes in the $80-\mathrm{M}_{\odot}$ ZAMS model. The top and the bottom panels are for strange modes.

radiation and $L_{r}=L$, where $P_{\mathrm{G}}$ and $P_{\mathrm{R}}$ stand for gas pressure and radiation pressure, respectively. The term proportional to $\kappa L$ comes from the gradient of radiation pressure. When this term is comparable to unity, the outward density decrease is hindered and a zone with a flat density distribution is produced. In such a zone, radiation pressure is dominant so that $V(\propto \rho / P)$ and hence $\omega_{\mathrm{c}}$ are depressed. The higher the ratio $L / M$, the thicker the zone, because the term proportional to $\kappa L$ becomes of order unity at a smaller $r / R$.

In the propagation diagram for an $\mathrm{HdC}$ model (lower panel of Fig. 4) a pulsation mode apparently cannot be trapped in the exterior propagation region because the evanescent zone around $r / R \sim 0.85$ is both too weak and too close to the surface (according to the run of $\omega_{t}$ ) in the appropriate range of frequencies. This may explain why adiabatic pulsations of helium stars do not show evidence of strange mode phenomena. The cause of the difference of the trapping between the two stellar model series can be attributed to the fact that the density at the opacity peak in ZAMS models is larger than at the second helium ionization zone in HdC models (see Fig. 1) and hence the effect of density inversion is stronger in the ZAMS models.

\subsection{Strange modes in extremely non-adiabatic pulsations}

Fig. 2 shows that although strange modes do not appear in adiabatic eigenfrequencies for the $\mathrm{HdC}$ models, they do appear in nonadiabatic and NAR modal diagrams. In these cases, also, the peculiar frequency change with control parameter (effective temperature) can be understood as a confinement of amplitude of certain modes to the outermost regions, around the density

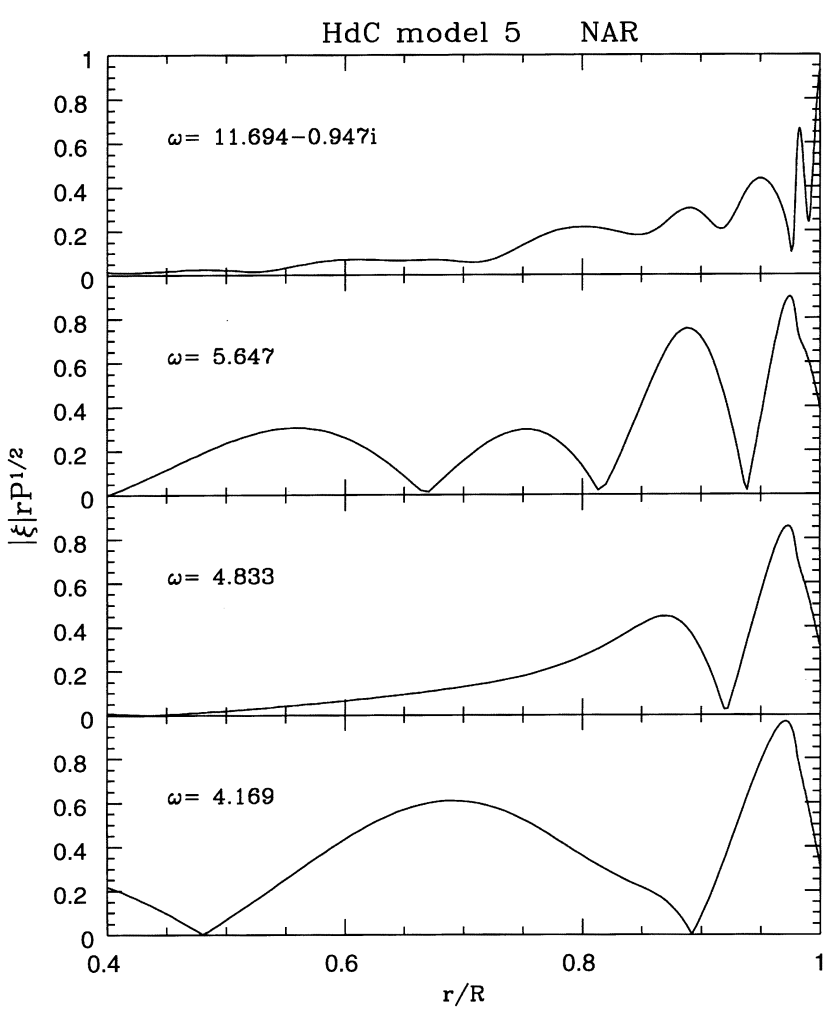

Figure 8. Runs of the amplitude of displacement in the NAR approximation for selected modes in the fifth $\mathrm{HdC}$ model. The top panel and the second panel from the bottom are for strange modes.

inversion, of the stellar models. The frequency of a strange mode in the $\mathrm{HdC}$ models increases with effective temperature, because the depth to a density inversion decreases.

Examples of the amplitude distribution of NAR modes in the $80-\mathrm{M}_{\odot}$ ZAMS model and the 5 th $\mathrm{HdC}$ model are given in Figs 7 and 8, respectively. In Fig. 7, the bottom and the top panels are for strange modes, while in Fig. 8 the second-bottom panel and the top panel are for strange modes. The density minimum is located at $r / R \simeq 0.96$ in the $80-\mathrm{M}_{\odot}$ ZAMS model (Fig. 1). Two density minima exist in the $\mathrm{HdC}$ model at $r / R \simeq 0.83$ and $\simeq 0.97$, which correspond, respectively, to the second and the first ionizations of helium. It is apparent from these figures that amplitudes of strange modes are considerably well confined to zones around the density inversions.

To estimate what plays an important role in the confinement of the amplitude of pulsation in the NAR approximation, we have obtained eigenfrequencies with artificially changing $\kappa_{T}, \kappa_{\rho}$, and $\chi_{T} / \chi_{\rho}$. Fig. 9 shows modal diagrams obtained in the condition of $\kappa_{T}=0$. The essential features of strange modes in the ZAMS models are preserved, but in the HdC models strange mode features have been strongly reduced. This figure indicates that the cause of amplitude confinement is different between ZAMS and HdC models. We furthermore note that the strength of the strange mode nature in modal diagrams is enhanced when $\kappa_{\rho}$ and/or $\chi_{T} / \chi_{\rho}$ are artificially increased.

In the following part of this subsection we investigate the cause of the confinement of the amplitude of strange modes in the NAR approximation. The cause of instability of strange modes in the NAR approximation will be discussed in Section 4 using a two-zone model. 

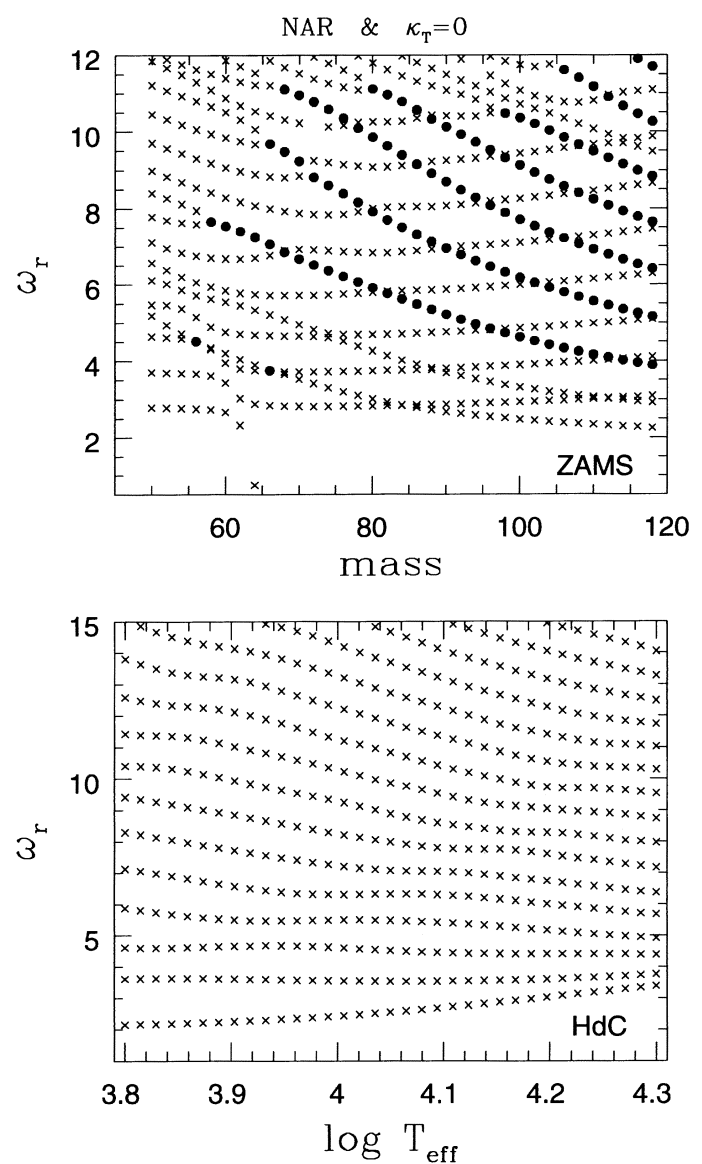

Figure 9. Modal diagrams obtained assuming $\kappa_{T}=0$ in the NAR approximation for ZAMS models (upper panel) and $\mathrm{HdC}$ models (lower panel). The meanings of the symbols are the same as in Fig. 2.

The linear pulsation equations in the NAR approximation may be written as

$$
\begin{aligned}
&\left(\sigma^{2}+4 \frac{G M_{r}}{r^{3}}\right) \rho \xi=\frac{\mathrm{d} \Delta P}{\mathrm{~d} r} \\
&=\frac{\mathrm{d}}{\mathrm{d} r}\left(P \chi_{\rho} \frac{\Delta \rho}{\rho}+P \frac{\chi_{T}}{4} \frac{\Delta P_{\mathrm{R}}}{P_{\mathrm{R}}}\right), \\
& \frac{\Delta \rho}{\rho}=-\frac{1}{r^{2}} \frac{\mathrm{d}}{\mathrm{d} r}\left(r^{2} \xi\right), \\
& \frac{\Delta L}{L}=4 \frac{\xi}{r}-\kappa_{\rho} \frac{\Delta \rho}{\rho}-\frac{\kappa_{T}}{4} \frac{\Delta P_{\mathrm{R}}}{P_{\mathrm{R}}}+\frac{1}{\left(\mathrm{~d} P_{\mathrm{R}} / \mathrm{d} r\right)} \frac{\mathrm{d} \Delta P_{\mathrm{R}}}{\mathrm{d} r}=0,
\end{aligned}
$$

where $\Delta$ indicates the Lagrangian perturbation of the variable just right of the symbol, $L$ is the radiative luminosity, and $P_{\mathrm{R}} \equiv a T^{4} / 3$ is the radiation pressure with $a$ being the radiation constant. The linearized equation of state

$\frac{\Delta P}{P}=\chi_{\rho} \frac{\Delta \rho}{\rho}+\frac{\chi_{T}}{4} \frac{\Delta P_{\mathrm{R}}}{P_{\mathrm{R}}}$

was used in the second equality in equation (4) (momentum equation). Equations (4)-(6) are, in general, reduced to a thirdorder differential equation of a single variable.

In order to understand the cause of the amplitude confinement of strange modes, it is instructive to derive a propagation diagram in the NAR approximation (such as those given in Fig. 4 for adiabatic pulsations). To do so we need to reduce the equation to a second-order differential equation: i.e. we need to make further simplifications. We consider two cases below.
Equations (4)-(6) are reduced to a second-order differential equation if we assume that $\left|\kappa_{\rho} \Delta \rho / \rho\right| \gg\left|\kappa_{T} \Delta P_{\mathrm{R}} / P_{\mathrm{R}}\right|$ and neglect the spatial change of $\chi_{T} P / P_{\mathrm{R}}$. This approximation is suitable for pulsation modes for which the temperature perturbation is much smaller than the density perturbation. We will call such pulsation modes quasi-isothermal ('QIT') modes. Since this approximation is similar to that of $\kappa_{T}=0$ (Fig. 9), we expect that QIT modes represent strange and ordinary modes in the ZAMS models, and ordinary modes in the $\mathrm{HdC}$ models.

Another way to reduce equations (4)-(6) to a second-order differential equation is to assume that the perturbation of radiation pressure is much larger than that of gas pressure. In this case the term $P \chi_{\rho} \Delta \rho / \rho$ in equation (4) is neglected. We will call such pulsation modes 'RPD' (radiation pressure dominant) modes.

We discuss these two types of modes now separately.

\subsubsection{QIT modes}

Neglecting the term $\kappa_{T} \Delta P_{\mathrm{R}} /\left(4 P_{\mathrm{R}}\right)$ in equation (6) and the spatial variation of $\chi_{T} P / P_{\mathrm{R}}$ in equation (4), we obtain

$\frac{\mathrm{d}^{2} \zeta}{\mathrm{d} r^{2}}+\frac{\zeta V}{r^{2} \chi_{\rho}} c_{1}\left(\omega^{2}-\omega_{\mathrm{QC}}^{2}\right)=0$

where

$\zeta=\xi r \sqrt{P \chi_{\rho}} \exp \left[\int \frac{\kappa_{\rho} \chi_{T}}{8 \chi_{\rho}} \frac{\mathrm{d} \ln P_{\mathrm{R}}}{\mathrm{d} r} \mathrm{~d} r\right]$,

$\omega_{\mathrm{QC}}^{2}=\frac{1}{c_{1}}\left[4 \chi_{T} \nabla_{T}-4+\frac{\chi_{\rho}}{V}\left(B^{2}+\frac{\mathrm{d} B}{\mathrm{~d} \ln r}-B\right)\right]$,

$B=\frac{1}{2}\left[\frac{\mathrm{d} \ln \left(P \chi_{\rho}\right)}{\mathrm{d} \ln r}-2+\frac{\kappa_{\rho} \chi_{T}}{4 \chi_{\rho}} \frac{\mathrm{d} \ln P_{\mathrm{R}}}{\mathrm{d} \ln r}\right]$,

$c_{1}=\left(\frac{r}{R}\right)^{3}\left(\frac{M}{M_{r}}\right)$.

Equation (7) indicates that QIT modes are propagative when $\omega^{2}>\omega_{\mathrm{QC}}^{2}$.

The spatial variations of $\omega_{\mathrm{QC}}$ in a ZAMS model and an HdC model are shown in Fig. 10 by long-dashed lines. There is a broad peak in $\omega_{\mathrm{QC}}$ around $r / R \sim 0.9$ in the ZAMS model. The peak separates inner and outer propagation zones for low-frequency modes. The cause of the peak is mainly a peak in $V$, i.e., essentially the same as for the adiabatic pulsations. By using a WKB analysis, we will show below that the peak of $\omega_{\mathrm{QC}}$ in ZAMS models can actually confine pulsation amplitude exterior to the peak. On the other hand, the spike of $\omega_{\mathrm{QC}}$ in the HdC model in Fig. 10, which is caused by the spike in $\nabla_{T}$, seems too thin to confine amplitude.

To see which modes in the full NAR calculations correspond to the QIT modes, we obtained eigenfrequencies of QIT modes by using a WKB approximation, i.e., we obtain $\omega$ from the relation

$\int_{a}^{b} \frac{1}{r} \sqrt{\frac{c_{1} V}{\chi_{\rho}}\left(\omega^{2}-\omega_{\mathrm{QC}}^{2}\right)} \mathrm{d} r=\left(n+\frac{1}{2}\right) \pi$,

where $a$ and $b$ represent the lower and the upper boundary of a propagation zone, and $n$ is a integer. For a given model, a sequence of frequencies are obtained corresponding to different values of $n$ $(=0,1, \ldots)$ for each propagation zone. The results are shown in Fig. 11 by open circles. The modal diagram for the QIT modes in our HdC models is very similar to that of the ordinary modes shown in Fig. 2 without any amplitude confinement feature. 

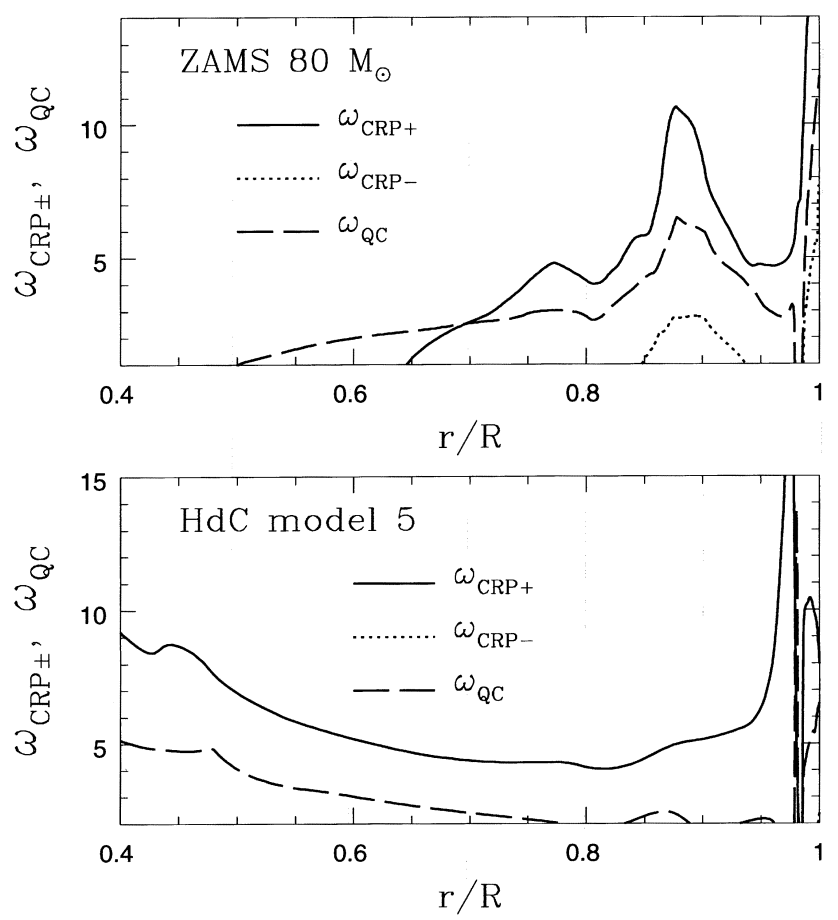

Figure 10. Runs of critical frequencies in the $80-\mathrm{M}_{\odot}$ ZAMS model (upper panel) and in the fifth $\mathrm{HdC}$ model (lower panel). Solid lines are for $\omega_{\mathrm{CRP}+}$ and dotted lines for $\omega_{\mathrm{CRP}-}$. The dotted line does not appear in the $\mathrm{HdC}$ model and appears only in small parts in the ZAMS model. The RPD oscillations are propagative when $\omega_{\mathrm{CRP}-}<\omega<\omega_{\mathrm{CRP}+}$. Thick-dashed lines give the critical frequency $\omega_{\mathrm{QC}}$, above which QIT oscillations are propagative.

For the massive ZAMS models, there are three types of sequences of eigenfrequencies. In the low-frequency range ( $\omega \lesssim 7$ : the boundary corresponds to the peak of $\omega_{\mathrm{QC}}$ in the upper panel of Fig. 10), two types of sequences appear. One type of sequences, for which frequencies slowly increase as the mass of the ZAMS increases, correspond to modes propagative interior to the peak of $\omega_{\mathrm{QC}}$. The location of the peak of $\omega_{\mathrm{QC}}$ shifts gradually inwards as the mass increases for the same reason given for the adiabatic pulsations (Section 3.1). The shift of the peak with mass decreases the thickness of the inner propagation zone. This explains the gradual increase of the frequencies with mass. The other type of sequences, for which frequencies decrease as the mass increases, correspond to modes confined in the outer propagation zone. The shift of the peak with mass causes gradual increase in the thickness of the outer propagation zone and decrease in the frequencies of modes trapped in it. The latter and the former types of sequences are similar to strange and ordinary modes, respectively, shown in Figs 2 and 9. This similarity clearly shows that the strange modes in the NAR approximation in the ZAMS models are nearly isothermal modes whose amplitude is confined around the density minimum just as in the adiabatic strange modes. For the sequences in the highfrequency range $(\omega \gtrsim 7)$, frequencies exceed the peak of $\omega_{\mathrm{QC}}$ so that they are propagative in most of the envelope. These modes seem to correspond to those seen in the upper part of the diagram for the ZAMS in Fig. 9.

To summarize the character of the QIT modes we note that they correspond to the ordinary modes in our HdC models and to the ordinary and the strange modes in our massive ZAMS models. This result is consistent with the fact that when $\kappa_{T}$ is artificially set to be zero, the strange modes disappear in the HdC models,
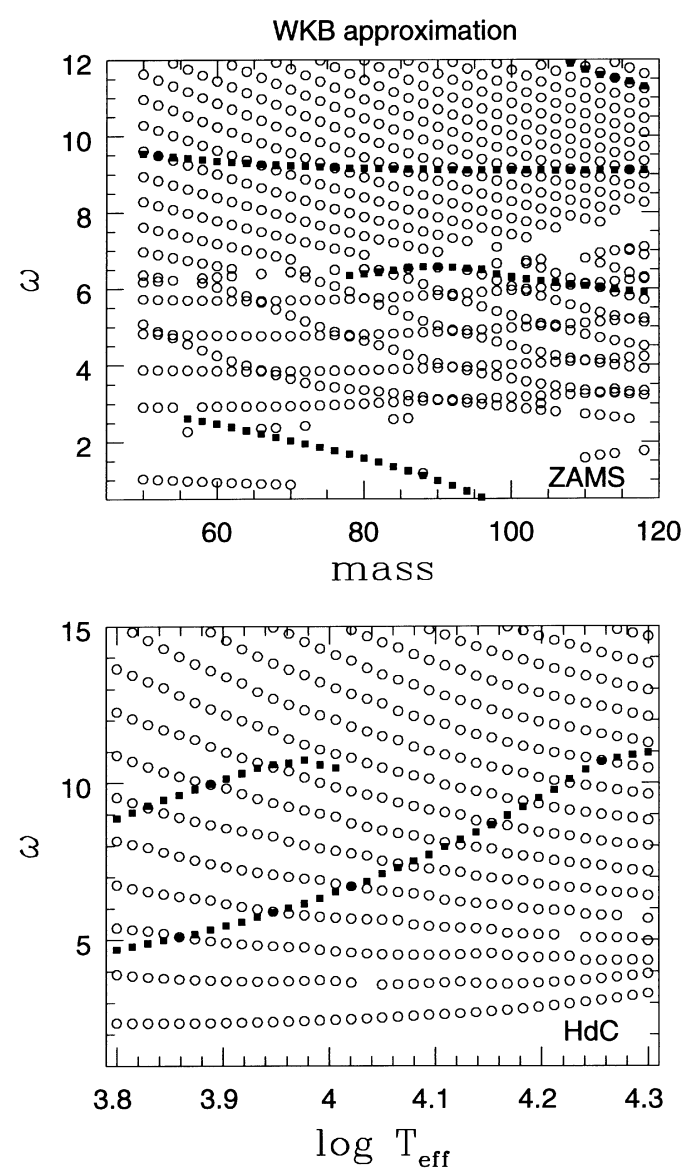

Figure 11. Modal diagrams obtained by using a WKB approximation for the ZAMS models (upper panel) and for the HdC models (lower panel). Open circles indicate QIT modes, and filled squares RPD modes.

but the modal diagram for the massive ZAMS models remains similar.

\subsubsection{RPD modes}

For RPD oscillations, we neglect the term $P \chi_{\rho} \Delta \rho / \rho$ in equation (4). From equations (4)-(6) we obtain, after some manipulations, a second-order differential equation:

$\frac{\mathrm{d}^{2} \eta}{\mathrm{d} r^{2}}+\frac{\eta}{r^{2}}\left[A_{0}+A_{1} \frac{c_{1} \omega^{2}}{q}-\left(\frac{c_{1} \omega^{2}}{2 q}\right)^{2}\right]=0$,

where

$$
\begin{aligned}
& \eta \equiv \xi r^{(1+h)} P^{1 / 2} \exp \left[-\frac{\omega^{2}}{2 q} \int^{r} \frac{c_{1}\left(r^{\prime}\right)}{r^{\prime}} \mathrm{d} r^{\prime}\right] \\
& A_{0} \equiv V\left[\frac{4\left(4-\kappa_{T}\right)}{\chi_{T} \kappa_{\rho}}+\frac{U}{2}+\frac{V}{4}+\frac{1}{2} \frac{\mathrm{d} \ln \rho}{\mathrm{d} \ln r}-2-\frac{2}{\kappa_{\rho}}-\frac{2}{q}\right] \\
&-(h+1)(h+2), \\
& A_{1} \equiv h+V\left(4-\kappa_{T}\right) \nabla_{T}+\frac{U-V}{2} \\
& q \equiv \chi_{T} \kappa_{\rho} \nabla_{T}, \\
& h \equiv 2\left(\chi_{T} \nabla_{T}-1\right) / q,
\end{aligned}
$$




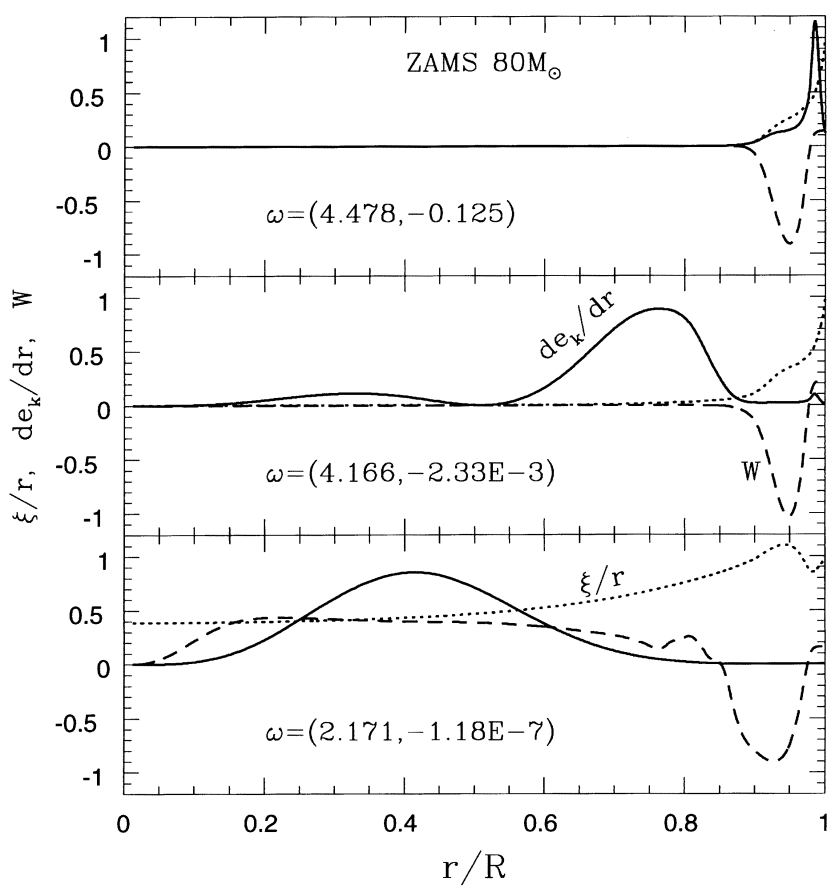

Figure 12. Eigenfunctions of the displacement ( $\xi / r$, dotted lines), pulsation energy distributions (solid lines) and cumulative work curves ( $W$, longdashed lines) for overstable modes in the $80-\mathrm{M}_{\odot}$ model in the full nonadiabatic analysis. If $\mathrm{d} W / \mathrm{d} r>0(\mathrm{~d} W / \mathrm{d} r<0)$ the zone acts to drive (damp) the pulsation. (The scale of the vertical axes is arbitrary.)

with $U \equiv \mathrm{d} \ln M_{r} / \mathrm{d} \ln r$. In deriving equation (10), we assumed that the spatial variations of $\chi_{T}, \nabla_{T}, \kappa_{T}$ and $\kappa_{\rho}$ are much smaller than the spatial pressure variation in the equilibrium envelope. We note here that if a plane-parallel approximation is employed, $A_{0}$ in equation (10) diminishes and the equation becomes similar to equation (5.6) in Glatzel (1994). Based on this equation, Glatzel argured that the strange modes correspond to high-frequency modes. We do not adopt his view, however, because modal diagrams show that both ordinary and strange modes exist in a similar frequency range.

Equation (10) indicates that RPD oscillations are propagative when

$\omega_{\mathrm{CRP}-}^{2}<\omega^{2}<\omega_{\mathrm{CRP}+}^{2}$,

where

$\omega_{\mathrm{CRP} \pm}^{2}=\frac{2 q}{c_{1}}\left(A_{1} \pm \sqrt{A_{1}^{2}+A_{0}}\right)$.

The spatial variations of $\omega_{\mathrm{CRP}+}$ and $\omega_{\mathrm{CRP}-}$ are shown in Fig. 10 by solid lines and dotted lines, respectively, for the ZAMS model of $80 \mathrm{M}_{\odot}$ and the fifth $\mathrm{HdC}$ model. The dotted line hardly appears in Fig. 10, because $\omega_{\text {CRP- }}^{2}$ is negative in most parts of the envelopes. This fact indicates that RPD modes with $\omega^{2}<0$ are possible. [The existence of such modes with pure imaginary eigenfrequencies was reported in the full non-adiabatic analysis of luminous helium star models with OPAL opacities by Saio (1995). It is interesting to note that the amplitude of such a mode was strongly confined in a zone where the contribution of gas pressure is a minimum, suggesting that the mode is a pure RPD mode.]

To see which modes in the full NAR calculations correspond to the RPD modes, we obtain eigenfrequencies using a WKB approximation, i.e., we obtain eigenfrequencies using the relation

$\int_{a}^{b} \frac{1}{r} \sqrt{A_{0}+A_{1} \frac{c_{1} \omega^{2}}{q}-\left(\frac{c_{1} \omega^{2}}{2 q}\right)^{2}} \mathrm{~d} r=\left(\frac{1}{2}+n\right) \pi$,

where $a$ and $b$ are, respectively, the lower and the upper boundaries of the propagation zone of RPD modes, and $n$ is a integer. The results are shown by filled squares in Fig. 11. For HdC models, we have two sequences that roughly mimic the two strange mode sequences in the full NAR calculations shown in Fig. 2. The lower frequency sequence corresponds to the modes trapped between around the density minimum due to the second helium ionization and the gap of the propagation zone located at the steep density peak at the first helium ionization zone. (The gap disappears if $\kappa_{T}$ is artificially set to zero.) The upper frequency sequence corresponds to modes trapped in the two propagation zones separated by the gap located at the steep density peak. Apparently, RPD modes correspond to strange modes in our HdC models.

Four sequences of RPD modes appear in the massive ZAMS models. In the $80-\mathrm{M}_{\odot}$ model the propagation range for each mode is as follows: $0.92 \lesssim r / R \lesssim 0.99$ for the mode of $\omega \simeq 1.57$, $0.98 \leqq r / R \lesssim 1.0$ for the mode of $\omega \simeq 6.39,0.87 \lesssim r / R \leqq 0.90$ for the mode of $\omega \simeq 9.15$, and $0.99 \lessgtr r / R \lesssim 1.0$ for the mode of $\omega \simeq 14.2$. The lowest sequence in Fig. 11 may correspond to the sequence of the neutrally stable strange modes whose frequency is about 1.9 at $80 \mathrm{M}_{\odot}$ in the NAR modal diagram in Fig. 2. It is hard to find other correspondence between a WKB RPD mode sequence and a feature in the modal diagram from the full NAR analysis for massive ZAMS models.

To summarize the results of RPD modes, we note that they are identified as strange modes in the HdC models, but they do not have an apparent relation with the unstable strange modes in the ZAMS models in the NAR limit.

\section{STABILITY OF STRANGE MODES}

Fig. 2 shows that stability properties of strange modes in full nonadiabatic analyses for a massive ZAMS are significantly different from those in the NAR approximation. (The difference is small in HdC models.) Different mechanisms seem to work in the stability of strange modes in these two cases. We will discuss them separately in the following.

\subsection{Strange modes in full non-adiabatic analyses}

For massive ZAMS models, the fully non-adiabatic pulsation frequencies of low-order modes, except for the lowest stable strange mode, are similar to the adiabatic frequencies (see Fig. 2). This suggests that non-adiabaticity is weak for these modes and that the overstability of some of them is induced by quasi-adiabatic processes such as $\kappa$ - and $\epsilon$-mechanisms (Glatzel \& Kiriakidis 1993a). In the full non-adiabatic analysis for ZAMS models some of the strange modes are pulsationally unstable and regular modes approaching unstable strange modes also tend to be overstable.

Fig. 12 shows cumulative work $W(r)$ (long-dashed lines) as well as amplitudes and pulsation energy distributions of the overstable modes in the $80-\mathrm{M}_{\odot}$ ZAMS model. Zones having positive (negative) $\mathrm{d} W / \mathrm{d} r$ drive (damp) pulsations. This figure shows that the trapping of the strange mode (uppermost panel) around the density inversion tends to quench the positive radiative damping in the lower part of the non-adiabatic region and to emphasize the driving in the outer part. A similar effect is seen in an adjacent regular mode near a crossing. In this case it appears that a partial reflection of the 


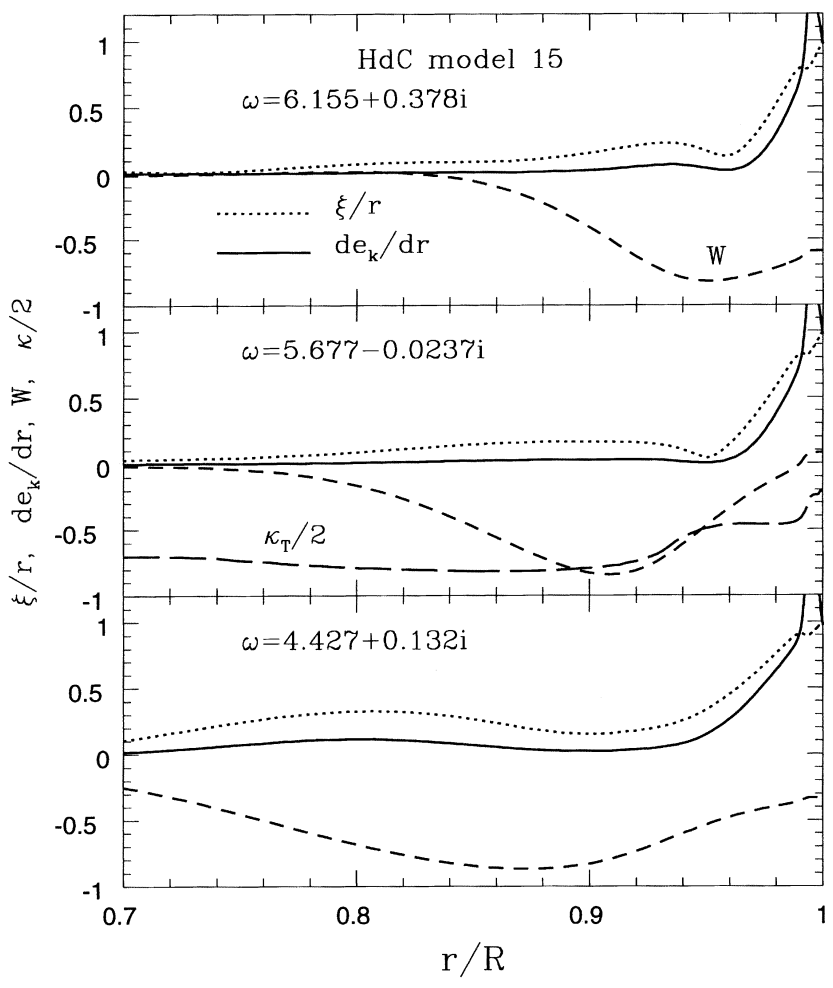

Figure 13. Work curves and amplitude distributions for a strange mode (middle panel) and its adjacent modes in full non-adiabatic analysis for the 15th HdC model. The opacity derivative $\kappa_{T}$ is also shown in the middle panel.

wave at the density inversion leads to a relative increase in amplitude in the driving region. The ratio of the imaginary to the real part of the eigenfrequency is much larger for the overstable strange mode than for the neighbouring regular mode. The nonadiabatic driving and damping are comparable in the two cases, but in the strange mode the total kinetic energy is very much diminished by being strongly confined to the outer layers. (The ratio of the imaginary to the real part of the eigenfrequency is proportional to the ratio of the non-adiabatic damping or driving energy to the kinetic energy of a pulsation mode.) By analogy to the adiabatic case, illustrated in Fig. 3, the ordinary mode looks much like the 10 mode illustrated there while the trapping of the kinetic energy in a strange mode is like that seen in the adiabatic $2 \mathrm{O}$ mode.

The fact that the $\kappa$-mechanism is responsible for the overstability of strange modes in the full non-adiabatic analysis of massive ZAMS models was confirmed by performing stability analyses with $\kappa_{T}$ being artificially set to be zero. In such analyses all the modes became damped although the modal diagram remained similar.

Fig. 12 shows that the $\epsilon$-mechanism hardly affects the stability of pulsation modes except for the lowest order mode, for which the cumulative work $W$ increases outward appreciably in the central nuclear burning region $(r / R \leqq 0.15)$. This mode corresponds to the fundamental mode in the adiabatic approximation. The limited influence of the $\epsilon$-mechanism in our massive ZAMS models was further confirmed by performing stability analyses with the $\epsilon$ mechanism artificially cut off. The only difference occurred in the modes with $\omega_{r} \simeq 2.1$, which correspond to the fundamental modes in the adiabatic approximation. They were overstable for
$M \geq 96 \mathrm{M}_{\odot}$ in this experimental analysis, while in the full analysis these modes are overstable for $M \geq 76 \mathrm{M}_{\odot}$ (Fig. 2). The stability properties of the other modes remained the same as in the full analyses.

Fig. 13 shows work curves as well as amplitudes and kinetic energy distributions for a strange mode and its adjacent modes in the full non-adiabatic analysis for the 15th $\mathrm{HdC}$ model ( $\log T_{\text {eff }} \simeq 4.005$ ). In this model one of the strange mode sequences crosses an ordinary mode sequence (see the right middle panel in Fig. 2). (In the NAR limit the model lies in the middle of an instability band brought about by the crossing of a strange mode sequence and an ordinary mode sequence.) The middle panel is for the strange mode. This figure shows that driving for ordinary modes does not necessarily occur where $\kappa_{T}$ increases outwards, contrary to weakly non-adiabatic pulsations in our massive ZAMS models, indicating that pulsations in the full non-adiabatic analysis of our $\mathrm{HdC}$ models are indeed very non-adiabatic. The uppermost panel is for the mode whose frequency is slightly higher than that of the strange mode and that becomes the complex conjugate counterpart of the overstable strange mode in the NAR limit. A part of the driving zone for the strange mode acts as a damping zone for the mode in the uppermost panel $(0.9 \lesssim r / R \lesssim 0.95)$. The excitation mechanism of overstable strange modes seems to change continuously from the $\kappa$-mechanism enhanced by the amplitude confinement in the weakly non-adiabatic condition to the mechanism in the NAR limit, which we discuss below.

\subsection{Stability of strange modes in the NAR limit}

In this subsection we discuss the cause of the strange mode instability in the extremely non-adiabatic limit, by using a twozone model in the plane-parallel approximation.

\subsubsection{Basic equations in the plane-parallel approximation}

We consider an outer thin zone in a stellar envelope. Let $r_{0}$ be the distance from the centre at the bottom of the zone. We introduce the height $z$ from the bottom, i.e., $r=r_{0}+z$. We also use a mass coordinate $m$ defined as

$\mathrm{d} m=\rho \mathrm{d} z \quad$ so that $\quad \mathrm{d} M_{r}=4 \pi r_{0}^{2} \mathrm{~d} m$.

Then the basic equations are

$\ddot{z}=-\frac{\partial P}{\partial m}-g$

$\frac{\partial z}{\partial m}=\frac{1}{\rho}$

$F=-\frac{4 a c}{3 \kappa} T^{3} \frac{\partial T}{\partial m}=-\frac{c}{\kappa} \frac{\partial P_{\mathrm{R}}}{\partial m}$,

where $g$ is the gravitational acceleration, $F$ is the radiation flux and $P_{\mathrm{R}} \equiv a T^{4} / 3$ the radiation pressure. Because the pressure $P$ is separated into the sum of gas pressure $P_{\mathrm{G}}$ and radiation pressure $P_{\mathrm{R}}$, we may write equation (14) in the following form:

$\ddot{z}=-\frac{\partial P_{\mathrm{G}}}{\partial m}+\frac{F \kappa}{c}-g$,

where equation (16) is used for $\partial P_{\mathrm{R}} / \partial m$. We note here that $F_{\kappa} / c=-\partial P_{\mathrm{R}} / \partial m$ represents the radiation force per unit mass. Gas pressure increases outward $\left(\mathrm{d} P_{\mathrm{G}} / \mathrm{d} m>0\right)$ when $F \kappa / c>g$ in an equilibrium structure, which produces a density inversion. 
Taking the Lagrangian perturbations of equations (14)-(16), and neglecting non-linear terms of the perturbed quantities, we obtain

$-\sigma^{2} \Delta z=-\frac{\partial\left(\Delta P_{\mathrm{G}}+\Delta P_{\mathrm{R}}\right)}{\partial m}$,

$\frac{\partial \Delta z}{\partial m}=-\frac{\Delta \rho}{\rho^{2}}$

$\frac{\Delta F}{F}=-\frac{\kappa_{T}}{4} \frac{\Delta P_{\mathrm{R}}}{P_{\mathrm{R}}}-\kappa_{\rho} \frac{\Delta \rho}{\rho}-\frac{c}{\kappa F} \frac{\partial \Delta P_{\mathrm{R}}}{\partial m}=0$,

where $\Delta$ indicates the Lagrangian perturbation of the quantity just at the right-hand side of the symbol. The perturbed quantities are assumed to be proportional to exp(i $\sigma t)$. Equation (19) represents the NAR condition, the extremely non-adiabatic limit. We assume, for simplicity, that the gas pressure obeys the ideal gas law:

$\frac{\Delta P_{\mathrm{G}}}{P_{\mathrm{G}}}=\frac{\Delta \rho}{\rho}+\frac{1}{4} \frac{\Delta P_{\mathrm{R}}}{P_{\mathrm{R}}}$.

\subsubsection{Two-zone model}

In order to understand the instability mechanism of strange modes, we employ here a simple two-zone model. The lower zone is bounded by $z_{0}$ and $z_{1}$ and the upper zone by $z_{1}$ and $z_{2}$, the stellar surface coincides with $z_{2}$. Both zones have the same thickness $\mu$ in terms of the mass coordinate $m$. We define $P_{\mathrm{G}}, P_{\mathrm{R}}, \rho$ at the middle of each zone. We write these quantities as $P_{\mathrm{G} i}, P_{\mathrm{R} i}, \rho_{i}$, with $i=1$ and 2 for the lower and upper zones, respectively. We write equations (17) to (19) as

$$
\begin{aligned}
& \sigma^{2} \Delta z_{1}=\left(\Delta P_{\mathrm{G} 2}-\Delta P_{\mathrm{G} 1}+\Delta P_{\mathrm{R} 2}-\Delta P_{\mathrm{R} 1}\right) / \mu, \\
& \sigma^{2} \Delta z_{2}=-2\left(\Delta P_{\mathrm{G} 2}+\Delta P_{\mathrm{R} 2}\right) / \mu, \\
& \frac{\Delta z_{1}}{\mu}=-\frac{\Delta \rho_{1}}{\rho_{1}^{2}}, \\
& \frac{\Delta z_{2}-\Delta z_{1}}{\mu}=-\frac{\Delta \rho_{2}}{\rho_{2}^{2}}, \\
& \frac{\kappa_{T 1}}{8} \frac{\Delta P_{\mathrm{R} 1}}{P_{\mathrm{R} 1}}+\frac{\kappa_{T 2}}{8} \frac{\Delta P_{\mathrm{R} 2}}{P_{\mathrm{R} 2}}+\frac{\kappa_{\rho}}{2}\left(\frac{\Delta \rho_{1}}{\rho_{1}}+\frac{\Delta \rho_{2}}{\rho_{2}}\right) \\
& \quad+\frac{c}{\kappa F \mu}\left(\Delta P_{\mathrm{R} 2}-\Delta P_{\mathrm{R} 1}\right)=0, \\
& \frac{\kappa_{T 2}}{4} \frac{\Delta P_{\mathrm{R} 2}}{\Delta P_{\mathrm{R} 2}}+\kappa_{\rho} \frac{\Delta \rho_{2}}{\rho_{2}}-\frac{2 c}{\kappa F \mu} \Delta P_{\mathrm{R} 2}=0,
\end{aligned}
$$

where we have used the fact that $\Delta P$ vanishes at the surface. Furthermore, we have neglected the spatial variation of $\kappa$ and $\kappa_{\rho}$.

From equations (21)-(26) and equation (20) applied to each zone separately, we obtain

$\sigma^{4} \mu^{4}-\sigma^{2} \mu^{2} \Psi+2 \Phi_{1} \Phi_{2}=0$

where

$\Psi=\Phi_{1}+3 \Phi_{2}-2 \frac{\rho_{2} f_{1} Q \kappa_{\rho}}{s_{1} s_{2}}$

with

$Q=\frac{\mu F \kappa}{2 c}$,

$\Phi_{i}=\rho_{i}\left(P_{\mathrm{G} i}+Q \kappa_{\rho} \frac{f_{i}}{s_{i}}\right)$,
$s_{i}=1-\frac{Q \kappa_{T i}}{4 P_{\mathrm{R} i}} \quad$ and $\quad f_{i}=1+\frac{P_{\mathrm{G} i}}{4 P_{\mathrm{R} i}}$

for $i=1,2$. We note that the value of $Q$ roughly represents the value of $P_{\mathrm{R}}$ (equation 16).

The property of the solutions of equation (27) for $\sigma^{2}$ is determined by the sign of the discriminant

$D=\Psi^{2}-8 \Phi_{1} \Phi_{2}$.

When $D<0, \sigma^{2}$ s are complex, while $\sigma^{2}$ s are real when $D>0$. It can be shown that the coefficient $\Psi$ and the discriminant $D$ are positive when $\kappa_{\rho}=0$. In this case, equation (27) has two positive solutions for $\sigma^{2}$, which means that all the pulsation frequencies are purely real. No strange mode instability occurs in this case. In other words, a necessary condition for strange mode instability (in the planeparallel configuration) is that $\kappa_{\rho}$ is positive. This property is consistent with our experience in obtaining eigenfrequencies with artificially changing opacity derivatives.

The cause of the relation between $\kappa_{\rho}$ and the strange mode instability can be understood from equation (19). If $\kappa_{\rho}$ is set to be zero in equation (19), we have a homogeneous equation for $\Delta P_{\mathrm{R}}$ which is decoupled from other pulsation variables. In this case, $\Delta P_{\mathrm{R}}=0$, i.e., the pulsation is isothermal. It can be shown quite generally that the pulsation frequency is purely real in the NAR approximation if the pulsation is isothermal.

In most parts of a stellar envelope, $\Phi_{i}$ values as well as $\Psi$ are positive, because $\kappa_{T}$ has a negative value or a small positive value except in a narrow ionization zone. Therefore, the discriminant can be negative (i.e., indicating strange mode instability) when $Q \kappa_{\rho}$ is large. The value of $Q(=\mu F \kappa / 2 c)$ roughly represents radiation pressure, and a larger $Q$ tends to produce a flat density distribution and density inversion [see equation $\left(14^{\prime}\right)$ ]. This is consistent with our numerical results that the strange mode instability occurs when the pulsation is trapped in an outer zone with a density inversion, and that the boundary of the strange mode instability in the Hertzsprung-Russell (HR) diagram is horizontal (i.e., $L=$ constant, which is lower for higher metallicity).

We also note that if $\kappa_{T}$ is positive, $s_{i}$ is less than unity and helps to make the discriminant negative. As seen in the modal diagram with $\kappa_{T}=0$ (Fig. 9), this effect is important in the HdC models. Actually, in the $\mathrm{HdC}$ models $\kappa_{T}>0$ around the broad density inversion at the second helium ionization zone (see Fig. 1). Therefore, the strange mode instability in the $\mathrm{HdC}$ models is mainly caused by the fact that the amplitude is confined in a zone in which $\kappa_{T}$ is large. This effect is not apparent in the ZAMS models because $P_{\mathrm{R}} / P_{\mathrm{G}}(=1 / \beta)$ is much higher.

As a simple example giving a negative value of $D$, let us assume that $\kappa_{T}=0$ (Fig. 9 indicates that strange modes in massive ZAMS models can be unstable even when $\kappa_{T}$ is artificially set to be zero) and $f_{1}=f_{2}=1$. The latter equality means that the two zones are dominated by radiation pressure. In this case

$\Phi_{i}=\rho_{i}\left(P_{\mathrm{G} i}+Q \kappa_{\rho}\right) \simeq \rho_{i} Q \kappa_{\rho}$

and hence the discriminant becomes

$D \simeq\left(Q \kappa_{\rho} \rho_{1}\right)^{2}\left[1-6\left(\rho_{2} / \rho_{1}\right)+\left(\rho_{2} / \rho_{1}\right)^{2}\right]$.

This equation indicates that $D$ is negative when $0.2 \lessgtr\left(\rho_{2} / \rho_{1}\right) \lessgtr 5.8$. This example shows that $D$ can actually be negative, depending on the mechanical structure of the star, and that the existence of a density inversion is not a necessary condition for the occurrence of strange mode instability. This is consistent with Papaloizou et al.'s (1997) finding that the strange mode instability occurs even in models with constant $\beta$ (no density inversion) if $\beta$ is sufficiently 
small. In our models, the density peak and the exterior to it seem to play an important role in confining amplitude by forming an evanescent zone.

A simple physical explanation of the instability occurring in the NAR limit can be derived using the plane-parallel formulae (17)-(19). Let us assume $\kappa_{T}=0$ for simplicity. Then equation (19) can be written as

$\frac{\partial \Delta P_{\mathrm{R}}}{\partial m}=-\frac{\kappa_{\rho} \kappa F}{c} \frac{\Delta \rho}{\rho}$.

When the envelope is compressed $(\Delta \rho>0), \partial \Delta P_{\mathrm{R}} / \partial m<0$; i.e., the outward force due to the gradient of radiation pressure increases, which produces a restoring force. (In other words, when matter is compressed, opacity increases due to $\kappa_{\rho}$. To compensate for this effect and keep $\Delta F=0$, the radiation pressure gradient is steepened.) Since the relation in equation (31) is not algebraic, the phase of $\Delta P_{\mathrm{R}}$ can be different from that of $\Delta \rho$ so that the restoring force can cause excitation or damping of pulsations. For oscillations propagating as $\exp (\mathrm{i} k r)$ the left-hand side of equation (31) is proportional to $i k \Delta P_{\mathrm{R}}$. The sign of the wavenumber $k$ determines the sign of the phase difference between $\Delta P_{\mathrm{R}}$ and $\Delta \rho$ as well as the direction of the propagation. This means that growing and damping oscillations (which appear as a pair in the NAR limit) correspond to the different directions of the propagation. (Glatzel 1994 argued this effect by referring to a work integral.) This restoring force is important only in a zone where radiation pressure dominates. Therefore, it can excite or damp only strange modes, the amplitude of which is confined to the outer part of the envelope.

As mentioned above, positive $\kappa_{T}$ plays an important role for the strange mode instability in the HdC models. This can be interpreted as follows. When matter is compressed, temperature generally increases. The temperature increase causes an increase in opacity if $\kappa_{T}>0$, which enhances the restoring force due to the steepening of the radiation pressure gradient. Apparently, how $\kappa_{T}$ contributes to strange mode instability is quite different from in the classical $\kappa$ mechanism.

\section{CONCLUSIONS}

We have discussed the properties of strange modes, which appear in the pulsation of stars with high luminosity-to-mass ratios. The strange modes are identified as pulsation modes whose frequencies change rapidly as a parameter of the model changes. In the extremely non-adiabatic condition, they often appear as pairs of strongly overstable and strongly damped modes.

The most important factor for the appearance of the strange modes is the confinement of pulsation amplitude to a small surface region, including density inversion, where radiation pressure is much larger than gas pressure. As a model parameter (such as the total mass or the effective temperature) varies, the location of the density inversion changes relative to the surface, which modifies the frequencies of strange modes. For a weak non-adiabatic condition, strange modes tend to be overstable due to the $\kappa$-mechanism because the amplitude confinement reduces the radiative damping in the inner part of the envelope. On the other hand, extremely nonadiabatic strange modes become overstable because the perturbation of radiation force (gradient of radiation pressure) provides a restoring force that can be out of phase with the displacement of pulsation. Using a simple two-zone model with the NAR approximation, which corresponds to the extremely non-adiabatic limit, we have found that a positive $\kappa_{\rho}$ is essential for the strange mode instability and that the effect is enhanced if $\kappa_{T}$ is positive. Although the opacity derivatives (especially $\kappa_{\rho}$ ) are important for the strange mode instability for the extremely non-adiabatic condition, the instability mechanism is different from the classical $\kappa$-mechanism.

In the ZAMS models, the amplitude confinement of strange modes both in the adiabatic and in the NAR approximations is mainly caused by the existence of a zone where the density gradient is weak and the ratio $P_{\mathrm{R}} / P_{\mathrm{G}}$ is very large. The strange modes trapped in such a zone can be overstable in the NAR limit as far as $\kappa_{\rho}>0$ due to the perturbation of radiation force as mentioned above.

In our HdC star models, no adiabatic strange modes appear. The mechanical structure seems not to be strong enough to confine the pulsation amplitude because the density around density inversions is much smaller than in the massive ZAMS models. The density inversions in the HdC models, for which we used Los Alamos opacities, exist at the first and the second helium ionizations. Strange modes appear in the non-adiabatic analysis and in the NAR approximation of the HdC models. Pulsations in HdC envelopes are extremely non-adiabatic and therefore they are intrinsically close to the pulsations in the NAR limit. Since the ratio $P_{\mathrm{R}} / P_{\mathrm{G}}$ is not so large as in the massive ZAMS models, the zone around density inversion where $\kappa_{T}$ is positive contributes significantly to the occurrence of the strange mode instability in the $\mathrm{HdC}$ models.

\section{ACKNOWLEDGMENT}

AG is grateful for financial support by the Swiss National Science Foundation.

\section{REFERENCES}

Aikawa T., 1985, Ap\&SS, 116, 401

Beyer H. R., 1995, J. Math. Phys., 36, 4815

Buchler J. R., Yecko P. A., Kolláth Z., 1997, A\&A, 326, 669

Cox J. P., 1974, Rep. Prog. Phys., 37, 563

Cox J. P., King D. S., Cox A. N., Wheeler J. C., Hansen C. J., Hodson S. W., 1980, Space Sci. Rev., 27, 531

Gautschy A., 1992a, MNRAS, 259, 82

Gautschy A., 1992b, A\&A, 260, 175

Gautschy A., 1993, MNRAS, 265, 340

Gautschy A., 1995, A\&A, 302, 401

Gautschy A., Glatzel W., 1990, MNRAS, 245, 597

Glatzel W., 1994, MNRAS, 271, 66

Glatzel W., Kiriakidis M., 1993a, MNRAS, 262, 85

Glatzel W., Kiriakidis M., 1993b, MNRAS, 263, 375

Glatzel W., Kiriakidis M., Fricke K. J., 1993, MNRAS, 262, L7

Iglesias C. A., Rogers F. J., Wilson B. G., 1992, ApJ, 397, 717

Kiriakidis M., Fricke K. J., Glatzel W., 1993, MNRAS, 264, 50

Kiriakidis M., Glatzel W., Fricke K. J., 1996, MNRAS, 281, 406

Papaloizou J. C. B., Alberts F., Pringle J. E., Savonije G. J., 1997, MNRAS, 284,821

Saio H., 1995, MNRAS, 277, 1393

Saio H., Jeffery C. S., 1988, ApJ, 714

Saio H., Wheeler J. C., Cox J. P., 1984, ApJ, 281, 318

Unno W., Osaki Y., Ando H., Saio H., Shibahashi H., 1989, Nonradial Oscillations of Stars, 2nd edn. Univ. Tokyo Press, Tokyo

Wood P. R., 1976, MNRAS, 156, 461

Worrell J. K., 1986, MNRAS, 174, 531

Zalewski J., 1992, PASJ, 44, 27

This paper has been typeset from a $\mathrm{T}_{\mathrm{E}} \mathrm{X} / \mathrm{L}^{\mathrm{A}} \mathrm{T}_{\mathrm{E}} \mathrm{X}$ file prepared by the author. 